\title{
CD38-Directed Therapies for Management of Multiple Myeloma
}

\author{
Hamza Hashmi' \\ Muhammad Husnain ${ }^{2}$ \\ Ali Khan ${ }^{3}$ \\ Saad Z Usmani ${ }^{3}$ \\ 'Department of Medicine, Division of \\ Hematology/Oncology, Medical \\ University of South Carolina, Charleston, \\ SC, USA; ${ }^{2}$ Department of Medicine, \\ Division of Hematology/Oncology, \\ University of Arizona, Tucson, AZ, USA; \\ ${ }^{3}$ Division of Plasma Cell Disorders, \\ Levine Cancer Institute/Atrium Health, \\ Charlotte, NC, USA
}

\begin{abstract}
The survival outcomes for multiple myeloma have improved several-fold in the past two decades, primarily due to the introduction of therapies with novel mechanisms of action including immunomodulatory agents, proteasome inhibitors, stem cell transplant and monoclonal antibodies in the schema of therapy. Antibody-based therapies targeting the surface marker CD38, namely daratumumab and isatuximab, have emerged as being highly effective as single agents as well as in combination regimens for both newly diagnosed and relapsed settings. Herein, the authors summarize the most recent data with both the current and emerging CD38-directed therapies in multiple myeloma.
\end{abstract}

Keywords: multiple myeloma, daratumumab, isatuximab, CD38, bispecific antibody, antibody drug conjugate

\section{Introduction}

Multiple myeloma is a hematological malignancy characterized by neoplastic proliferation of clonal plasma cells. ${ }^{1}$ Several landmark advances have been made in the treatment landscape of multiple myeloma in the last several decades. The use of high-dose chemotherapy followed by autologous hematopoietic stem cell transplant in the eligible patients as well as the approval of novel agents such as proteasome inhibitors (PI), immunomodulatory drugs (IMiD) and monoclonal antibodies in the last several decades have substantially improved the survival outcomes of myeloma patients. With the use of these novel strategies, an increasing proportion of patients are able to achieve a long-lasting remission. However, a vast majority of patients eventually have disease relapses and are often resistant to the previously used agents. $^{2}$ This raises the need to further advance the therapeutic armamentarium for patients with multiple myeloma, exploring novel mechanisms.

Targeted immunotherapy with antibodies directed against specific antigens on the surface of cancer cells has become one of the most promising treatment options for some cancers. ${ }^{3}$ In this regard, CD38 is a valuable surface antigen target in multiple myeloma due to the high expression of CD38 on their cell surface and preclinical studies demonstrating its potential immunosuppressive effect in the tumor microenvironment, promoting immune escape. ${ }^{4}$

CD38 is a transmembrane glycoprotein encoded by a gene on chromosome $4 .^{5}$ CD38, as a molecule, is composed of a long C-terminal extracellular domain, a transmembrane segment, and a short N-terminal cytoplasmic tail. CD38 has a variable expression during various stages of development and differentiation of lymphocytes. While there is a high expression of CD38 on medullary thymocytes,
Correspondence: Saad Z Usmani Clinical Research in Hematologic Malignancies, Levine Cancer Institute/ Atrium Health, Charlotte, NC, USA $\mathrm{Tel}+1$ 980-442-4363

Email saad.usmani@atriumhealth.org 
this expression is diminished in the majority of mature $\mathrm{T}$ cells till their reactivation in the immune cascade. ${ }^{6}$ Similarly, for B lymphocytes, CD38 expression is upregulated in the bone marrow precursor cells, diminished in resting (normal) $\mathrm{B}$ cells, and enhanced in terminally differentiated plasma cells. The CD38 molecule is highly expressed in both normal as well as malignant plasma cells (greater than 90\%). ${ }^{7}$

The CD38 expression is also seen on other cells of the hematopoietic stem cell system including natural killer cells $(60 \%)$ and monocytes $(60 \%)$. Alternatively, neutrophils, red blood cells, and platelets have an absent to low CD38 expression. ${ }^{8}$ More importantly, CD38 expression is also noticed on regulatory $\mathrm{T}$ cells, regulatory $\mathrm{B}$ cells and myeloid derived suppressor cells with a high surface expression of CD38 associated with compromised immune surveillance for malignancies ${ }^{9}$ (Figure 1, panel A). The consistently high expression of CD38 on malignant plasma cells has prompted the development of targeted immunotherapies. The mechanism of action of anti-CD38 agents comprises of antibody dependent cell mediated cytotoxicity (ADCC), antibody dependent cell mediated phagocytosis (ADCP), complement dependent cytotoxicity (CDC) as well as immunomodulatory effects in the tumor microenvironment ${ }^{10,11}$ (Figure 1, panel B). Here, we discuss how the discovery and targeting of CD38 by different agents has revolutionized the treatment landscape of multiple myeloma.

\section{Anti-CD38 Naked Antibodies}

Daratumumab is a humanized IgG1 monoclonal antiCD38 naked antibody. Daratumumab showed significant antibody and complement dependent cytotoxicity (ADCC, $\mathrm{CDC}$ ) and FcR mediated crosslinking and apoptosis in preclinical studies. ${ }^{10,12}$ This led to a phase 1 clinical trial, by Lokhorst et al, that investigated the safety and efficacy of single agent daratumumab in patients with relapsed/refractory multiple myeloma. The overall response rate (ORR) was $36 \%$ in the $16 \mathrm{mg} / \mathrm{kg}$ cohort with median progression free survival (PFS) of 5.6 months. Sixty-four percent of the patients had double refractory disease; ie refractory to both PI and IMiD, $79 \%$ of the patients had disease that was refractory to the last therapy received and $76 \%$ of the patients had received prior autologous stem cell transplant. Grade 3 or 4 adverse events were mainly pneumonia and cytopenias. About $40 \%$ of the patients had serious adverse events, which were mainly infection-related events. ${ }^{13}$ On the basis of this study, an effective dose of daratumumab was determined to be $16 \mathrm{mg} / \mathrm{kg}$. This was followed by a phase 2 study by Lonial et al, which included patients with double refractory disease (refractory to both PI and IMiDs) or who had disease progression after 3 or more lines of therapies. The ORR was $29.2 \%$ with a median PFS of 3.7 months and a median duration of response of 7.4 months. ${ }^{14}$ Based on these encouraging results, single agent daratumumab was approved by the Federal Drug Administration (FDA) in patients with relapsed/refractory multiple myeloma patients who had received at least 3 prior lines of therapy or who were double refractory to a PI and an IMiD.

These encouraging results of single agent daratumumab were then followed by combination studies of daratumumab with lenalidomide and bortezomib. A phase 3 study (POLLUX) randomized 569 patients with relapsed/ refractory multiple myeloma to the combination of daratumumab, lenalidomide and dexamethasone (DRd) versus lenalidomide and dexamethasone (Rd) in a 1:1 fashion. After a median follow up of 44 months, PFS in the DRd arm was 44.5 months as compared to 17.5 months in the $\mathrm{Rd}$ arm. Minimal residual disease (MRD) negativity was seen in $30 \%$ of the patients in the DRd arm as compared to $5.3 \%$ in the $\mathrm{Rd}$ arm. ${ }^{15}$ The study clearly demonstrated the superiority of triplets over doublets as well as deep responses seen with addition of daratumumab to lenalidomide-dexamethasone backbone. In the "real world" clinical practice, this study is relevant in Europe where not all patients are lenalidomide exposed or refractory at the time of disease relapse. Another similar study CASTOR trial randomized 498 patients with relapsed/refractory multiple myeloma to daratumumab in combination with bortezomib and dexamethasone (DVd) versus bortezomib and dexamethasone (Vd). Median PFS at 3 years was 16.7 vs 7.1 months in DVd vs Vd arm respectively. ${ }^{16}$ MRD negativity was $15.1 \%$ in the DVd arm as compared to $1.6 \%$ in the $\mathrm{Vd}$ arm. ${ }^{17}$ This study is relevant in the United States as DVd is both effective and safe for patients who relapse on lenalidomide based maintenance therapy with prior exposure but not refractoriness to PIs. Patients with lenalidomide and bortezomib refractory multiple myeloma present a highly refractory group of patients with limited options. CANDOR was a phase III randomized controlled trial that enrolled patients who were either refractory or exposed to lenalidomide and or bortezomib. A total of 466 patients were randomized to either daratumumab, carfilzomib and dexamethasone (DKd) versus carfilzomib and 


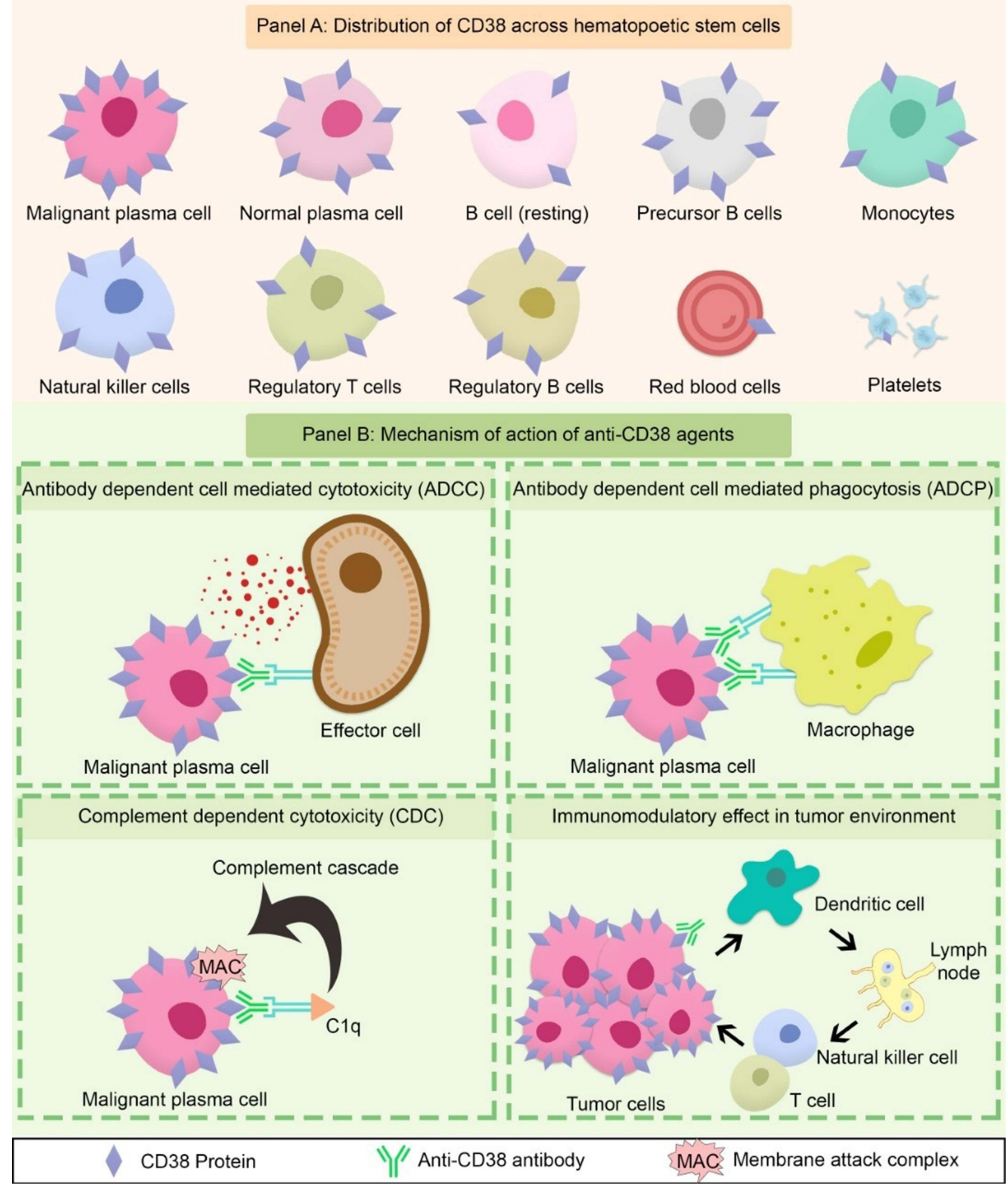

Figure I Panel (A) distribution of CD38 across hematopoietic stem cells; Panel (B) mechanism of action of anti-CD38 agents.

dexamethasone $(\mathrm{Kd})$. About $42 \%$ patients were lenalidomide exposed, $90 \%$ were bortezomib exposed, $33 \%$ were lenalidomide refractory and $29 \%$ were bortezomib refractory. Median PFS is still not reached in the DKd arm versus 15.8 months in the $\mathrm{Kd} \mathrm{arm},{ }^{18}$ making $\mathrm{DKd}$ a desirable option to achieve deep and durable responses for patients with high risk cytogenetic abnormalities that may benefit from addition of second-generation PI to 
anti-CD38 monoclonal antibody at the time of first relapse. Daratumumab in combination with pomalidomide and dexamethasone (DPd) was studied in patients with relapsed/refractory multiple myeloma after 2 or more lines of therapies showing an overall response in $60 \%$ of the patients. The study also showed a median PFS of 8.8 months and an OS of 17.5 months in the treated patient population. ${ }^{19} \mathrm{DPd}$ was studied in another phase II study in patients with lenalidomide exposed or refractory multiple myeloma patients showing an ORR of $77 \%$ with 1 -year PFS of $75 \% .^{20}$ APOLLO is a phase 3 trial which randomized 304 multiple myeloma patients with relapsed/refractory disease to receive either subcutaneous daratumumab in combination with pomalidomide and dexamethasone (DPd) versus Pd. Median PFS for DPd was 12.4 months versus 6.9 months for Pd arm, ${ }^{21}$ making DPd another favorable salvage chemotherapy option for patients who may not be able to tolerate carfilzomib due to age/frailty and/or cardiac dysfunction. It is important to highlight that given differences in patient populations, number of prior lines of therapy, and previous exposure/refractoriness to lenalidomide/bortezomib; cross trial comparisons cannot be drawn amongst these different anti-CD38 monoclonal antibody-based regimens.

Encouraging results in the relapsed refractory setting prompted the evaluation of daratumumab in the frontline setting for newly diagnosed multiple myeloma. In the ALCYONE trial, a total of 706 newly diagnosed transplant ineligible patients were randomized to daratumumab in combination with bortezomib, melphalan and prednisone (D-VMP) versus bortezomib, melphalan and prednisone (VMP) arms. The ORR was 91\% in the D-VMP arm as compared to $74 \%$ in the VMP arm, 18-month PFS was $71.6 \%$ in D-VMP arm as compared to $50.2 \%$ in the VMP group with median PFS not reached in the D-VMP arm with HR of $0.50,95 \%$ CI of $0.38-0.65, \mathrm{p}<0.001$. Minimal residual disease (MRD) negativity was $22.3 \%$ in the D-VMP group as compared to $6.2 \%$ in the VMP group. The estimated 36-month overall survival was 78\% in the D-VMP group and $67.9 \%$ in the VMP group $(95 \% \mathrm{CI}$ 73.2-82.0). ${ }^{22}$ The MAIA trial was another phase 3 trial that randomized 737 newly diagnosed, transplant ineligible patients to DRd or Rd groups. After a median follow up of about 28 months, median PFS for the DRd arm was not reached as compared to 31.9 months for the $\mathrm{Rd}$ arm (HR 0.56, 95\% CI, 0.43-0.73, p < 0.001). About 25\% of the patients were able to achieve MRD negative disease in the DRd group as compared to only $7.3 \%(\mathrm{p}<0.001)$ in the $\mathrm{Rd}$ group. ${ }^{23}$ Based on the encouraging safety and efficacy outcomes from these first line studies, daratumumab based triplet therapy is now routinely being used in the upfront setting for treatment of the newly diagnosed transplant ineligible patients with multiple myeloma.

In the transplant eligible patient population, CASSIOPEIA trial randomized a total of 1085 newly diagnosed transplant eligible multiple myeloma patients to receive either daratumumab, bortezomib, thalidomide and dexamethasone (D-VTD) or bortezomib, thalidomide and dexamethasone (VTD). The primary end point of the trial was stringent complete response (sCR) at day +100 post autologous stem cell transplant. At day +100 post autologous stem cell transplant, $29 \%$ of the patients in the Dara-VTD arm had achieved stringent complete response as compared to $20 \%$ in the VTD arm. At 18 months follow up, median PFS in the D-VTD arm was $93 \%$ as compared to $85 \%$ in the VTD arm (HR $0.47,95 \%$ CI $0.33-0.67, p<0.0001)$. Sixty-four percent of the patients achieved MRD negativity in the D-VTD arm as compared to $44 \%$ in the VTD arm. ${ }^{24}$ The GRIFFIN study randomized 292 multiple myeloma patients with newly diagnosed disease to either daratumumab in combination with lenalidomide, bortezomib, and dexamethasone (D-RVD) versus lenalidomide, bortezomib and dexamethasone (RVD). Primary endpoint was sCR which was seen in $42.4 \%$ of patients in the D-RVD group versus $32 \%$ in the RVD group ( $p<0.068$ ). About $50 \%$ of patients achieved MRD negative disease in the D-RVD group as compared to only $20 \%$ in the RVD group ( $p<0.0001$ ), at a median follow up of 22.1 months. ${ }^{25}$ The daratumumab based quadruplet has shown significant efficacy in another phase II trial in combination with carfilzomib, lenalidomide and dexamethasone (D-KRD) in transplant eligible newly diagnosed multiple myeloma patients, with MRD negative rate of $83 \%{ }^{26}$ The MASTER trial is currently studying D-KRD induction followed by autologous stem cell transplantation and response adapted D-KRD consolidation in newly diagnosed multiple myeloma patients. Patients will receive 0,4 or 8 cycles of consolidation depending on the MRD status. For the 38 treated patients with evaluable data, rates of MRD negativity were $34 \%$, $70 \%$ and $80 \%$ after induction, transplant and at best response, respectively. ${ }^{27}$ PERSEUS is an ongoing randomized phase 3 randomized controlled trial studying a combination of daratumumab, bortezomib, lenalidomide and dexamethasone versus bortezomib, lenalidomide and dexamethasone in newly diagnosed transplant eligible 
patients (Clinical Trials. Gov Identifier, NCT03710603). This trial will help guide our decision to use quadruplet versus triplet therapy in newly diagnosed multiple myeloma patients that are considered transplant eligible. Daratumumab is currently being studied in different combinations in the newly diagnosed transplant eligible, transplant ineligible, post-transplant maintenance and relapsed/ refractory multiple myeloma patients. Results of these studies will change the way we treat multiple myeloma in coming years.

Isatuximab is a new anti-CD38 humanized IgG1 monoclonal antibody, which is being studied extensively in multiple myeloma. In preclinical studies, isatuximab has shown anti-myeloma activity which can be mediated by many different mechanisms including ADCC, CDC, ADCP and pro-apoptotic activity, via FcR crosslinking. ${ }^{28}$ Feng et al showed that isatuximab targets CD38 T regulatory cells hence restoring immune function in human multiple myeloma cell lines. ${ }^{29}$ Moreno et al showed that isatuximab could sensitize CD38 (high expression) multiple myeloma cells to bortezomib and dexamethasone. While ADCC was seen against both CD38 high and low expression multiple myeloma cells, ADCP was seen only in CD38 high expression multiple myeloma cells, and complement dependent cytotoxicity was seen in less than half of patient samples. ${ }^{30}$ Encouraging anti-myeloma activity of isatuximab in xenograft models of multiple myeloma led to a first-in-human, phase 1 study of isatuximab in relapsed refractory multiple myeloma to determine the safety and efficacy of isatuximab monotherapy. A total of 84 patients were enrolled in the study. In patients receiving $10 \mathrm{mg} / \mathrm{kg}$ dose, the ORR was $23.8 \%$, including 1 complete remission (CR) with median PFS of 3.7 months. About $50 \%$ of the patients experienced infusion related reactions, mostly grade $1-2 .{ }^{31}$ Martin et al assessed the efficacy and safety of isatuximab in combination with lenalidomide and dexamethasone in patients with relapsed/refractory multiple myeloma in a phase $1 \mathrm{~b}$ study. The ORR with the combination was $52 \%$, associated with a median PFS of 8.5 months. ${ }^{32}$ Isatuximab was also studied in combination with pomalidomide and dexamethasone for patients with relapsed/ refractory multiple myeloma who had received 2 prior lines of therapies. Patients received isatuximab at 5, 10 or $20 \mathrm{mg} / \mathrm{kg}$ (weekly for 4 weeks, followed by every 2 weeks), pomalidomide $4 \mathrm{mg}$ (days 1-21) and dexamethasone $40 \mathrm{mg}$ weekly in a 28 -days cycle. The combination yielded an ORR of about $60 \%$ with a median PFS of 17.6 months. ${ }^{33}$ Dose of $10 \mathrm{mg} / \mathrm{kg}$ was chosen as standard for all future studies. Attal et al randomized 307 multiple myeloma patients with relapsed refractory disease to isatuximab in combination with pomalidomide and dexamethasone (Isa-Pd) or pomalidomide and dexamethasone (Pd) arms. Median PFS was 11.5 months in the IsaPd arm as compared to 6.5 months in the Pd arm (HR $0.56,95 \%$ CI $0.44-0.81 ; \mathrm{p}<0.001) .{ }^{34}$ Moreau et al randomized 332 patients with relapsed/refractory multiple myeloma to a combination of isatuximab, carfilzomib and dexamethasone (Isa-Kd) versus carfilzomib and dexamethasone $(\mathrm{Kd})$ showing an ORR of $86.6 \%$ in the Isa-Kd arm as compared to $82.9 \%$ in the $\mathrm{Kd}$ arm $(\mathrm{p}<0.19)$. Median PFS was not reached in the Isa-Kd arm as compared to 19.1 months in the Kd arm (HR 0.53, 99\% CI $0.32-0.89, \mathrm{p}<0.0007) .{ }^{35}$ These encouraging results have established isatuximab in combination with $\mathrm{Pd}$ or $\mathrm{Kd}$ as an acceptable alternative to daratumumab in patients with relapsed/refractory disease.

More recently, Mikhael et al published a phase 2 study of isatuximab monotherapy in patients with heavily pretreated multiple myeloma who were previously refractory to daratumumab. ${ }^{36} \mathrm{~A}$ total of 32 patients were enrolled who had received a median of 7 prior lines of therapy and all were refractory to daratumumab. More than half [60\%] had received daratumumab as the last line of therapy, prior to isatuximab. One (3\%) patient achieved minimal response while $17(50 \%)$ achieved stable disease with a disease control rate [defined as minimal response or stable disease $\geq 8$ weeks] of $37.5 \%$. Median PFS was 1.6 months and median OS was 10.7 months. The disease control rate doubled in patients with a washout period (between the last dose of daratumumab and first dose of isatuximab) greater than 6 months $(58 \%)$ versus patients with a washout period less than 3 months (28.6\%). Although there were no objective responses, more than half the patients achieved stable disease as best overall response, with the longest duration of stable disease being 18.5 months. This study raises the need to further explore the optimal timing and sequencing of these different CD38 targeting monoclonal antibodies in patients with relapsed refractory multiple myeloma. Several ongoing trials are investigating the role of various isatuximab combinations in newly diagnosed and relapsed/refractory multiple myeloma patients (Table 1).

Anti-CD38 naked antibodies appear to have a tolerable toxicity profile with infusion related reactions (IRR) as the main side effect. Intravenous daratumumab and 
Table I Selected Trials of Isatuximab in Multiple Myeloma

\begin{tabular}{|c|c|c|c|}
\hline Clinical Trial & Phase & Setting & Treatment \\
\hline NCT036I773I & 3 & Newly diagnosed & Isa+VRD, \\
\hline (GMMG HD7) & & transplant eligible & $\mathrm{R}$ maintenance \\
\hline NСT03319667 & 3 & Newly diagnosed & Isa+VRD \\
\hline (IMROZ) & & transplant ineligible & \\
\hline NCT03I 04842 & 2 & $\begin{array}{l}\text { Newly diagnosed } \\
\text { high risk }\end{array}$ & Isa+KRD \\
\hline NCT025I3I86 & I & $\begin{array}{l}\text { Newly diagnosed } \\
\text { transplant ineligible }\end{array}$ & Isa+VCD \\
\hline NCT04083898 & $\mathrm{I} / 2$ & Relapsed/refractory & Isa+BP \\
\hline NCT03194867 & $\mathrm{I} / 2$ & Relapsed/refractory & Isa+cemiplimab \\
\hline NCT02332850 & I & Relapsed/refractory & Isa+K \\
\hline
\end{tabular}

Abbreviations: Isa, isatuximab; VRD, bortezomib, lenalidomide and dexamethasone; KRD, carfilzomib, lenalidomide and dexamethasone; VCD, bortezomib, cyclophosphamide and dexamethasone; BP, Bendamustine and prednisone; K, carfilzomib.

isatuximab are associated with IRRs in about $50 \%$ of the patients, mostly grade $1 / 2$, comprising of respiratory symptoms including nasal congestion, cough, throat irritation and dyspnea. ${ }^{15-19,33-36}$ They mostly occur during the first infusion and are infrequent after the second infusion. IRRs are managed with prophylaxis with a combination of antihistamine, corticosteroid, leukotriene receptor antagonist, and temporary interruption of the infusion. ${ }^{37}$ The incidence of IRR is significantly reduced with a subcutaneous formulation of daratumumab. ${ }^{38} \mathrm{CD} 38$ targeting antibodies can be detected as monoclonal protein on serum protein electrophoresis and immunofixation and hence interfere with evaluation of response. Daratumumab-specific immunofixation reflex assay can be used to distinguish the therapeutic versus disease related M protein. ${ }^{39}$ Similarly, CD38 naked antibodies bind to CD38 on red blood cells causing a pan-reactive indirect antiglobulin test on antibody screens. This requires red blood cell phenotyping or genotyping in patients prior to the initiation of CD38 antibody treatment. Despite interference with blood typing and cross matching, no adverse events associated with blood transfusion have been reported. ${ }^{40}$

\section{CD38 Antibody Drug Conjugates}

The remarkable success of CD38-directed naked monoclonal antibodies have encouraged the development of conjugated and bispecific antibodies. Antibody drug conjugates (ADCs) are monoclonal antibodies that are attached to a cytotoxic compound directed against surface antigens on the targeted cells. These antibodies specifically target cells that express their antigen of interest (target antigen). Once internalized, the cytotoxic payload is released through lysosome degradation, and ultimately leads to cell death. This targeted delivery of the cytotoxic compound spares the non-malignant healthy cells that lack the cell surface antigen, which in turn helps to limit offtarget toxicity. ${ }^{41}$

The payload is a cytotoxic chemical with potent activity that exerts its effect by interruption of the cell cycle by microtubule inhibition and DNA damage. Maytansinoid derivatives including DM1 (emtansine and mertansine) and DM4 (soravtansine and ravtansine) as well as auristatin derivatives including monomethyl-auristatin E (MMAE, vedotin) and monomethyl auristatin $\mathrm{F}$ (MMAF, mafodotin) are such payloads that are microtubule inhibitors. ${ }^{42}$ Duocarmycin and pyrrolobenzodiazepine dimers are calicheamicins that damage DNA. ${ }^{43}$ Several immunotoxins and radio-immunoconjugates coupled with the anti-CD38 monoclonal antibodies have shown a reasonable efficacy and safety profile in preclinical models.

Goldmacher et al showed that an immunotoxin consisting of an anti-CD38 antibody (HB7) coupled to a modified ricin molecule was capable of exerting potent cytotoxic activity against CD38 positive human lymphoma and myeloma cells with relatively low level of activity against other components of the hematopoietic system. ${ }^{44}$ Bolognesi et al reported an immunotoxin composed of an anti-CD38 monoclonal antibody (IB4) coupled to saporinS6 (a type 1 ribosome-inactivating protein), with effective targeted killing of CD38 positive human lymphoma cells while sparing other cell lines. ${ }^{45}$

Conventional radiotherapy has been used as a successful approach for solitary plasmacytomas as well as for palliation of symptoms in patients with extra medullary disease, highlighting the radio-sensitivity of malignant plasma cells. Anti-CD38 monoclonal antibodies can be used to deliver radionuclides to malignant plasma cells. Targeted radioimmunotherapy with Yttrium90 anti-CD38 monoclonal antibody was used by Green et al to optimize the delivery of a radioimmunotherapy conjugate. ${ }^{46}$ In a two-step process, first a construct composed of an antiCD38 antibody is administered and localizes to the CD38 receptors on the myeloma tumor cells. In the second step, a small molecular weight radioactive molecule (radioDOTA-biotin) is infused that binds to the antibody construct while the unbound radioactive molecules are excreted in the urine. This unique mechanism optimizes the delivery of radiotherapy to the cancer cells while 
minimizing exposure to the normal healthy cells, hence enhancing the therapeutic index with this novel approach. Advancements along similar lines led to the development of a bispecific anti-CD38/anti- ${ }^{90}$ Y-DOTA that has been evaluated in mice models. ${ }^{47}$ All of the mice treated with the construct achieved remission from multiple myeloma, with a low level of activity/toxicity seen in otherwise normal tissue. In similar pre-clinical models, the immunotoxin constructed by splicing the nanobody with a bacterial toxin (PE38), showed highly selective cytotoxicity against patient-derived multiple myeloma, as well as other cell lines, with half maximal effective concentration reaching as low as $10(-11)$ molar. The effectiveness of the immunotoxin could be further augmented by enhancing the CD38 expression on the cell surface using retinoic acid. ${ }^{48}$

\section{CD38 Bispecific T Cell Engagers in Multiple Myeloma}

Bispecific antibodies are able to simultaneously engage epitopes on the surface of target cells and $\mathrm{T}$ cells, thus bringing the $\mathrm{T}$ cells within physical proximity to kill the cancer cells. This novel mechanism of action has been applied in clinical practice by the use of blinatumomab (bispecific monoclonal antibody targeting CD3 on T cells and CD19 on leukemia cells) in patients with acute lymphoblastic leukemia. ${ }^{49}$ Currently, there are two bispecific antibodies targeting CD3 on T cells and CD38 on myeloma cells, namely AMG424 and GBR1342, that are being investigated in clinical trials. By simultaneously binding to CD38 expressed on cancer cells and CD3 expressed on $\mathrm{T}$ cells, these bispecific antibodies lead to T-cell activation and proliferation, releasing cytokines that ultimately kill CD38 positive cancer cells. ${ }^{8}$

GBR 1342 is a bispecific monoclonal antibody that targets CD3 on T cells and CD38 on myeloma cells where it has demonstrated the ability to direct $\mathrm{T}$ cells cytotoxicity towards myeloma cells. ${ }^{50}$ Based on the results of these preclinical studies, GBR 1342 is currently being investigated as monotherapy in highly pretreated relapsed/ refractory myeloma patients (NCT03309111).

Chu et al have also developed and tested a bispecific monoclonal antibody targeting CD3 on T cell and CD38 on myeloma cells. ${ }^{51}$ This monoclonal antibody comprises a Fc domain modified to eliminate the binding of Fc- $\gamma$ receptors, which helps to reduce non-selective activation of T cells. The presence of Fc allows for a long serum half-life overcoming a major limitation for other biphasic monoclonal antibodies requiring a continuous infusion via pump. This monoclonal antibody has been tested in preclinical studies where it not only eliminated human myeloma cells but was also able to engage effector T cells to kill CD38 positive cells in humanized mice and monkey models.

Cytokine release syndrome (CRS) might occur as a consequence of the $\mathrm{Fc}$ regions of bispecific antibodies binding Fc $\gamma$ receptors on $\mathrm{T}$ cells causing nonspecific activation of $\mathrm{T}$ cells. ${ }^{52}$ To mitigate the off-target toxicity of these bispecific T-cell engagers, a mutational Fc domain was introduced to the bispecific T-cell engagers. This strategy could help improve safety as well as the efficacy of these bispecific T-cell engagers. ${ }^{53}$

\section{CD38 CAR T-Cell Therapy in Multiple Myeloma}

The introduction of chimeric antigen receptor (CAR) T-cell therapy has revolutionized the treatment outcomes in patients with relapsed refractory acute lymphoblastic leukemia as well as non-Hodgkin's lymphoma. ${ }^{54}$

Advantages of CAR T-cell therapy over monoclonal antibodies are the ability of the CAR T-cells to produce potent antitumor responses and ability to persist for a long period of time exacting durable antitumor surveillance. However, given the potent efficacy, the choice of target antigen is of prime significance when compared to monoclonal antibodies due to the high risk of off-target toxicity. As CD38 is not only expressed in malignant plasma cells but also in many other cell types, this raises concerns about the safe use of anti-CD38 CAR T-cells in multiple myeloma patients. Several strategies have been developed to overcome excessive toxicity related to this novel therapy.

Drent and colleagues described a novel strategy aimed at enhancing the affinity of CD38 CAR T cells for myeloma cells, while sparing other CD38 positive cells of the hematopoietic system, thus improving the safety profile of CAR T-cells. ${ }^{55}$ The group reported the preclinical development of $\mathrm{T}$ cells equipped with an anti-CD38 CAR construct with an intracellular 4-1BB costimulatory domain. ${ }^{56}$ Upon engagement with the target antigen, these CAR T-cells were able to proliferate, produce inflammatory cytokines and cause lysis of multiple myeloma cell lines. The activity of the CAR T cells correlated with cell surface expression of CD38. Although; these CAR T-cells also targeted CD38 normal hematopoietic cells, CD34positive and CD38-negative progenitor cells were unharmed. 
A CAR T construct equipped with a suicide gene that can be triggered on demand, as a safety switch, has several notable applications. These include improving the safety of the adaptively transferred CAR T-cells, modulation of the cell surface expression of the receptors on the CAR T-cells, and optimization of the CAR affinity for its target to enhance selective killing. Along these lines, the safety and efficacy of a doxycycline-inducible-Tet design on antiCD38 CAR T-cells were tested by a Dutch group. ${ }^{57}$ The investigators showed that by using different doses of doxycycline, different levels of CAR expression could be induced at the cell surface, which could be used as a strategy to mitigate excessive toxicity. CAR-mediated lysis of the target cells is dependent on the dose of doxycycline and following the similar principle, the elimination of doxycycline leads to a successful cessation of off-tumor lysis. This novel mechanism, based upon optimization of the affinity of CAR T-cells directed against the CD38 target, allows the ability to differentiate between CD38 high myeloma cell lines and CD38 low-intermediate hematopoietic stem cells. These mechanisms used to optimize safety allow this approach to be an interesting exploration of efficacy in clinical practice.

Using the principles studied by Drent et al to reduce the on-target and off-tumor toxicity, ${ }^{55,57}$ Sorrento Therapeutics also released interesting pre-clinical data on an anti-CD38 CAR T-cell model. The antigen binding domain of the CAR T was composed of a fully humanized single chain variable fragment with variable binding affinities for the CD38 target antigen. The chimeric antigen receptor "selected" for transduction of the CAR T-cells had potent anti-tumor cytotoxicity at the cost of minimal off-tumor effect. Upon engagement with the target antigen in myeloma mice models, these CAR T-cells demonstrated the properties of activation, proliferation, and cytokine release that led to efficient killing of myeloma cells. While the anti-tumor effect was similar to the high affinity CAR T-cells, no activity was seen in cells with normal or low CD38 expression. An ongoing trial (NCT03464916) is the first study of this agent in human subjects.

\section{Future Directions}

Immunotherapeutic approaches have changed the treatment landscape for relapsed refractory multiple myeloma. Optimizing efficacy without compromising safety should be the future goals for the development of these immunotherapy-based treatments for patients with relapsed refractory multiple myeloma.
With its high expression on malignant hematological cancer cells, CD38 is a desirable cell surface target with novel antibody-based therapies. However, CD38 expression is also seen on other normal hematopoietic stem cells ie, monocytes, natural killer cells and $\mathrm{T}$ cells, although at an intermediate level. Hence, there is a need to optimize the affinity towards malignant plasma cells as well as minimize off-target toxicity towards other components of the hematopoietic system. Similarly, binding of the Fcy receptors on the $\mathrm{T}$ cells by the $\mathrm{Fc}$ region of the bispecific T-cell engagers causes nonspecific T-cell activation inducing significant side effects. This can be potentially mitigated by the use of the mutated $\mathrm{Fc}$ domain in the bispecific T-cell engagers to avoid the antigen dependent release of cytokines.

Another area of unmet need in improving personalized treatment with monoclonal antibodies is the development of laboratory assays and biomarkers that can readily and reliably predict the response to therapy. Given the high expression of CD38 seen in the early stages of plasma cell development and differentiation, anti-CD38 agents are being investigated for other plasma cell dyscrasias including smoldering myeloma to determine whether an early therapeutic intervention can prevent disease progression to symptomatic myeloma. It has also been observed that relapsed refractory multiple myeloma cell lines have either a diminished or complete loss of CD38 expression on the surfaces. This raises the need to assess CD38 expression prior to the initiation of therapy with anti-CD38 monoclonal antibodies.

Several mechanisms of resistance to antibody treatment have been proposed. Resistance to therapy may be directly related to the down-regulation of the CD38 antigen on the surface of the myeloma cell, especially in patients with an aggressive extra medullary relapse. Moreover, with increased levels of complement inhibitors (CD55, CD59) and antiapoptotic molecules, there is also upregulation of regulatory $\mathrm{T}$ cells promoting escape from immune surveillance. ${ }^{58}$ Although; these novel antibody-based therapies are highly effective in heavily pretreated patients, most patients eventually relapse. Sustaining the durability of response and preventing the emergence of resistant clones are important goals in the treatment landscape of relapsed refractory multiple myeloma. The pursuit of these goals will require use of combination of these novel antibody-based therapies with other chemo immunotherapies as well as use of CD38 based immunotherapy early on in disease course. An integrative approach 
consisting of genomic analysis, transcription mechanisms and drug metabolism is necessary to understand the underlying pathophysiology of resistance to immunotherapy and emergence of resistant clones leading to disease relapse.

\section{Conclusion}

With tolerable safety and a remarkable efficacy profile, use of anti-CD38 based immunotherapeutic approaches have expanded the treatment armamentarium for multiple myeloma. Optimizing efficacy without compromising safety, patient selection and timing of therapy, deeper understanding of drug resistance and strategies to mitigate treatment should be the future goals for the development of immunotherapy-based treatment for multiple myeloma.

\section{Disclosure}

H.H., M.H and A.K. have no conflicts to declare. S.Z.U. Research funding: Amgen, Array Biopharma, BMS, Celgene, GSK, Janssen, Merck, Pharmacyclics, Sanofi, Seattle Genetics, SkylineDX, Takeda; Consulting: Amgen, BMS, Celgene, EdoPharma, GSK, Janssen, Sanofi, Seattle Genetics, SecuraBio, SkylineDX, Takeda, TeneoBio; Speaking fees: Amgen, BMS, Janssen, Sanofi; Personal fees: Gilead, Genentech, outside the submitted work.

\section{References}

1. Palumbo A, Anderson K. Multiple myeloma. N Engl J Med. 2011;364 (11):1046-1060. doi:10.1056/NEJMra1011442

2. Kumar SK, Dispenzieri A, Lacy MQ, et al. Continued improvement in survival in multiple myeloma: changes in early mortality and outcomes in older patients. Leukemia. 2014;28(5):1122-1128. doi:10. 1038/leu.2013.313

3. Weiner LM. Building better magic bullets - improving unconjugated monoclonal antibody therapy for cancer. Nat Rev Cancer. 2007;7 (9):701-706. doi:10.1038/nrc2209

4. van de Donk NWCJ, Richardson PG, Malavasi F. CD38 antibodies in multiple myeloma: back to the future. Blood. 2018;131(1):13-29. doi:10.1182/blood-2017-06-740944

5. Nakagawara K, Mori M, Takasawa S, et al. Assignment of CD38, the gene encoding human leukocyte antigen CD38 (ADP-ribosyl cyclase/ cyclic ADP-ribose hydrolase), to chromosome 4p15. Cytogenet Cell Genet. 1995;69(1-2):38-39. doi:10.1159/000133933

6. Deaglio S, Mehta K, Malavasi F. Human CD38: a (r)evolutionary story of enzymes and receptors. Leuk Res. 2001;25(1):1-12. doi:10.1016/ S0145-2126(00)00093-X

7. Leo R, Boeker M, Peest D, et al. Multiparameter analyses of normal and malignant human plasma cells: $\mathrm{CD} 38++, \mathrm{CD} 56+, \mathrm{CD} 54+, \mathrm{cIg}+$ is the common phenotype of myeloma cells. Ann Hematol. 1992;64 (3):132-139. doi:10.1007/BF01697400

8. Atanackovic D, Steinbach M, Radhakrishnan SV, Luetkens T. Immunotherapies targeting $\mathrm{CD} 38$ in multiple myeloma. Oncoimmunology. 2016;5(11):e1217374. doi:10.1080/2162402X.20 16.1217374
9. Krejcik J, Casneuf T, Nijhof IS, et al. Daratumumab depletes CD38+ immune regulatory cells, promotes T-cell expansion, and skews T-cell repertoire in multiple myeloma. Blood. 2016;128(3):384-394. doi:10.1182/blood-2015-12-687749

10. de Weers M, Tai Y-T, van der Veer MS, et al. Daratumumab, a novel therapeutic human CD38 monoclonal antibody, induces killing of multiple myeloma and other hematological tumors. J Immunol. 2011;186(3):1840-1848. doi:10.4049/jimmunol.1003 032

11. Krejcik J, Frerichs KA, Nijhof IS, et al. Monocytes and granulocytes reduce CD38 expression levels on myeloma cells in patients treated with daratumumab. Clin Cancer Res. 2017;23(24):7498-7511. doi:10.1158/1078-0432.CCR-17-2027

12. Jansen JHM, Marco jansen JH, Boross P, et al. Daratumumab, a human CD38 antibody induces apoptosis of myeloma tumor cells via fc receptor-mediated crosslinking. Blood. 2012;120(21):2974. doi:10.1182/blood.v120.21.2974.2974

13. Lokhorst HM, Plesner T, Laubach JP, et al. Targeting CD38 with daratumumab monotherapy in multiple myeloma. $N$ Engl $\mathrm{J}$ Med. 2015;373(13):1207-1219. doi:10.1056/NEJMoa1506348

14. Lonial S, Weiss BM, Usmani SZ, et al. Daratumumab monotherapy in patients with treatment-refractory multiple myeloma (SIRIUS): an open-label, randomised, phase 2 trial. Lancet. 2016;387 (10027):1551-1560. doi:10.1016/S0140-6736(15)01120-4

15. Bahlis NJ, Dimopoulos MA, White DJ, et al. Daratumumab plus lenalidomide and dexamethasone in relapsed/refractory multiple myeloma: extended follow-up of POLLUX, a Randomized, Open-Label, Phase 3 Study. Leukemia. 2020;34(7):1875-1884. doi:10.1038/s41375-020-0711-6

16. Mateos M-V, Sonneveld P, Hungria V, et al. Daratumumab, bortezomib, and dexamethasone versus bortezomib and dexamethasone in patients with previously treated multiple myeloma: three-year follow-up of CASTOR. Clin Lymphoma Myeloma Leuk. 2020;20 (8):509-518. doi:10.1016/j.clml.2019.09.623

17. Avet-Loiseau H, San-Miguel J, Casneuf T, et al. Evaluation of sustained minimal residual disease negativity with daratumumab-combination regimens in relapsed and/or refractory multiple myeloma: analysis of POLLUX and CASTOR. $J$ Clin Oncol. 2021;39(10):JCO2001814. doi:10.1200/JCO.20.01814

18. Dimopoulos M, Quach H, Mateos M-V, et al. Carfilzomib, dexamethasone, and daratumumab versus carfilzomib and dexamethasone for patients with relapsed or refractory multiple myeloma (CANDOR): results from a randomised, multicentre, open-label, phase 3 study. Lancet. 2020;396(10245):186-197. doi:10.1016/S01 40-6736(20)30734-0

19. Chari A, Suvannasankha A, Fay JW, et al. Daratumumab plus pomalidomide and dexamethasone in relapsed and/or refractory multiple myeloma. Blood. 2017;130(8):974-981. doi:10.1182/blood-2017-05785246

20. Siegel DS, Schiller GJ, Samaras C, et al. Pomalidomide, dexamethasone, and daratumumab in relapsed refractory multiple myeloma after lenalidomide treatment. Leukemia. 2020;34(12):3286-3297. doi:10. 1038/s41375-020-0813-1

21. Dimopoulos MA, Terpos E, Boccadoro M, et al. Apollo: phase 3 randomized study of subcutaneous daratumumab plus pomalidomide and dexamethasone (D-Pd) versus pomalidomide and dexamethasone (Pd) alone in patients (Pts) with relapsed/refractory multiple myeloma (RRMM). Blood. 2020;136(Supplement 1):5-6. doi:10.1182/ blood-2020-135874

22. Mateos M-V, Dimopoulos MA, Cavo M, et al. Daratumumab plus bortezomib, melphalan, and prednisone for untreated myeloma. Transfus Cell Ther. 2018;378(6):518-528. doi:10.1056/nejmoa171 4678

23. Facon T, Kumar S, Plesner T, et al. Daratumumab plus lenalidomide and dexamethasone for untreated myeloma. N Engl J Med. 2019;380 (22):2104-2115. doi:10.1056/nejmoa1817249 
24. Roussel M, Moreau P, Hebraud B, et al. Bortezomib, thalidomide, and dexamethasone with or without daratumumab for transplantation-eligible patients with newly diagnosed multiple myeloma (CASSIOPEIA): health-related quality of life outcomes of a randomised, open-label, phase 3 trial. Lancet Haematol. 2020;7 (12):e874-e883. doi:10.1016/S2352-3026(20)30356-2

25. Voorhees PM, Kaufman JL, Laubach J, et al. Daratumumab, lenalidomide, bortezomib, and dexamethasone for transplant-eligible newly diagnosed multiple myeloma: the GRIFFIN trial. Blood. 2020;136(8):936-945. doi:10.1182/blood.2020005288

26. Landgren O, Hultcrantz M, Lesokhin AM, et al. Weekly carfilzomib, lenalidomide, dexamethasone and daratumumab (wKRd-D) combination therapy provides unprecedented MRD negativity rates in newly diagnosed multiple myeloma: a Clinical and Correlative Phase 2 Study. Blood. 2019;134(Supplement_1):862. doi:10.1182/blood2019-126378

27. Costa LJ, Chhabra S, Godby KN, et al. Daratumumab, carfilzomib, lenalidomide and dexamethasone (Dara-KRd) induction, autologous transplantation and post-transplant, response-adapted, measurable residual disease (MRD)-based dara-krd consolidation in patients with newly diagnosed multiple myeloma (NDMM). Blood. 2019;134(11):860. doi:10.1182/blood-2019-123170

28. Deckert J, Wetzel M-C, Bartle LM, et al. SAR650984, A novel humanized CD38-targeting antibody, demonstrates potent antitumor activity in models of multiple myeloma and other CD38 +hematologic malignancies. Clin Cancer Res. 2014;20(17):4574-4583. doi:10.1158/1078-0432.ccr-14-0695

29. Feng X, Zhang L, Acharya C, et al. Targeting CD38 suppresses induction and function of $\mathrm{t}$ regulatory cells to mitigate immunosuppression in multiple myeloma. Clin Cancer Res. 2017;23 (15):4290-4300. doi:10.1158/1078-0432.ccr-16-3192

30. Moreno L, Perez C, Zabaleta A, et al. The mechanism of action of the anti-CD38 monoclonal antibody isatuximab in multiple myeloma. Clin Cancer Res. 2019;25(10):3176-3187. doi:10.1158/1078-0432. ccr-18-1597

31. Martin T, Strickland S, Glenn M, et al. Phase I trial of isatuximab monotherapy in the treatment of refractory multiple myeloma. Blood Cancer J. 2019;9(4):41. doi:10.1038/s41408-019-0198-4

32. Martin T, Baz R, Benson DM, et al. A phase 1b study of isatuximab plus lenalidomide and dexamethasone for relapsed/refractory multiple myeloma. Blood. 2017;129(25):3294-3303. doi:10.1182/blood2016-09-740787

33. Mikhael J, Richardson P, Usmani SZ, et al. A phase 1b study of isatuximab plus pomalidomide/dexamethasone in relapsed/refractory multiple myeloma. Blood. 2019;134(2):123-133. doi:10.1182/blood2019-02-895193

34. Attal M, Richardson PG, Rajkumar SV, et al. Isatuximab plus pomalidomide and low-dose dexamethasone versus pomalidomide and low-dose dexamethasone in patients with relapsed and refractory multiple myeloma (ICARIA-MM): a randomised, multicentre, open-label, phase 3 study. Lancet. 2019;394(10214):2096-2107. doi:10.1016/S0140-6736(19)32556-5

35. Maiolino A, Moreau P, Dimopoulos M, et al. Isatuximab plus carfilzomib and dexamethasone vs carfilzomib and dexamethasone in relapsed/refractory multiple myeloma (IKEMA): interim analysis of a phase 3, Randomized, Open-Label Study. Hematol Transfus Cell Ther. 2020;42:262-263. doi:10.1016/j.htct.2020.10.439

36. Mikhael J, Belhadj-Merzoug K, Hulin C, et al. A phase 2 study of isatuximab monotherapy in patients with multiple myeloma who are refractory to daratumumab. Blood Cancer J. 2021;11(89). doi:10.1038/s41408-021-00478-4

37. Moreau P, van de Donk NWCJ, Miguel JS, et al. Practical considerations for the use of daratumumab, a novel CD38 monoclonal antibody, in myeloma. Drugs. 2016;76(8):853-867. doi:10.1007/ s40265-016-0573-4
38. Usmani SZ, Nahi H, Mateos MV, van de Donk N, Chari A. Subcutaneous delivery of daratumumab in relapsed or refractory multiple myeloma. Blood. 2019;134(8):668-677. doi:10.1182/ blood.2019000667

39. van de Donk NWCJ, Otten HG, El Haddad O. Interference of daratumumab in monitoring multiple myeloma patients using serum immunofixation electrophoresis can be abrogated using the daratumumab IFE reflex assay (DIRA). Clin Chem Lab Med. 2016;54 (6):1105-1109. PMID: 26812873. doi:10.1515/cclm-2015-0888.

40. Oostendorp M, Lammerts van Bueren JJ, Doshi P. When blood transfusion medicine becomes complicated due to interference by monoclonal antibody therapy. Transfusion. 2015;55(6 Pt 2):1555-1562. Epub 2015 May 18. PMID: 25988285. doi:10.1111/trf.13150

41. Tolcher AW. Antibody drug conjugates: lessons from 20 years of clinical experience. Ann Oncol. 2016;27(12):2168-2172. doi:10. 1093/annonc/mdw424

42. Nittoli T, Kelly MP, Delfino F, et al. Antibody drug conjugates of cleavable amino-alkyl and aryl maytansinoids. Bioorg Med Chem. 2018;26(9):2271-2279. doi:10.1016/j.bmc.2018.02.025

43. Peters C, Brown S. Antibody-drug conjugates as novel anti-cancer chemotherapeutics. Biosci Rep. 2015;35(4). doi:10.1042/bsr20150089

44. Goldmacher VS, Bourret LA, Levine BA, et al. Anti-CD38-blocked ricin: an immunotoxin for the treatment of multiple myeloma. Blood. 1994;84(9):3017-3025. doi:10.1182/blood.V84.9.3017.3017

45. Bolognesi A, Polito L, Farini V, et al. CD38 as a target of IB4 mAb carrying saporin-S6: design of an immunotoxin for ex vivo depletion of hematological CD38+ neoplasia. J Biol Regul Homeost Agents. 2005; 19(3-4):145-152.

46. Green DJ, Orgun NN, Jones JC, et al. A preclinical model of CD38-pretargeted radioimmunotherapy for plasma cell malignancies. Cancer Res. 2014;74(4):1179-1189. doi:10.1158/ 0008-5472.CAN-13-1589

47. Green DJ, Orgun NN, Hylarides MD, et al. Anti-CD38 pretargeted radioimmunotherapy demonstrates therapeutic efficacy in a human multiple myeloma mouse xenograft model. Blood. 2011;118 (21):1842. doi:10.1182/blood.v118.21.1842.1842

48. Li T, Qi S, Unger M, et al. Immuno-targeting the multifunctional CD38 using nanobody. Sci Rep. 2016;6(1):27055. doi:10.1038/ srep27055

49. Kantarjian H, Stein A, Gökbuget N, et al. Blinatumomab versus chemotherapy for advanced acute lymphoblastic leukemia. $N$ Engl J Med. 2017;376(9):836-847. doi:10.1056/NEJMoa1609783

50. Richter JR, Landgren CO, Kauh JS, et al. Phase 1, multicenter, open-label study of single-agent bispecific antibody t-cell engager GBR 1342 in relapsed/ refractory multiple myeloma. J Clin Oncol. 2018;36(15_suppl):TPS3132-TPS3132. doi:10.1200/jco.2018.36.15_suppl.tps 3132

51. Chu SY, Miranda Y, Phung S, et al. Immunotherapy with long-lived anti-CD38 $\times$ anti-CD3 bispecific antibodies stimulates potent T cellmediated killing of human myeloma cell lines and CD38 cells in monkeys: a potential therapy for multiple myeloma. Blood. 2014;124 (21):4727. doi:10.1182/blood.v124.21.4727.4727

52. Chatenoud L, Ferran C, Legendre C, et al. In vivo cell activation following OKT3 administration. Systemic cytokine release and modulation by corticosteroids. Transplantation. 1990;49(4):697-702. doi:10.1097/00007890-199004000-00009

53. Wang L, Hoseini SS, Xu H, Ponomarev V, Cheung N-K. Silencing Fc domains in $\mathrm{T}$ cell-engaging bispecific antibodies improves T-cell trafficking and antitumor potency. Cancer Immunol Res. 2019;7 (12):2013-2024. doi:10.1158/2326-6066.cir-19-0121

54. June $\mathrm{CH}$, Sadelain M. Chimeric antigen receptor therapy. $N$ Engl $J$ Med. 2018;379(1):64-73. doi:10.1056/NEJMra1706169

55. Drent E, Themeli M, Poels R, et al. A rational strategy for reducing on-target off-tumor effects of CD38-chimeric antigen receptors by affinity optimization. Mol Ther. 2017;25(8):1946-1958. doi:10.1016/ j.ymthe.2017.04.024 
56. Drent E, Groen RWJ, Noort WA, Themeli M, van Bueren JJL. Preclinical evaluation of $\mathrm{CD} 38$ chimeric antigen receptor engineered $\mathrm{T}$ cells for the treatment of multiple myeloma. Haematologica. 2016;101(5):616-625. doi:10.3324/haematol.2015.137620

57. Drent E, Poels R, Mulders MJ, et al. Feasibility of controlling CD38-CAR T cell activity with a Tet-on inducible CAR design. PLoS One. 2018;13(5):e0197349. doi:10.1371/journal.pone.0197349
58. Van de Donk NWCJ, Usmani SZ. CD38 antibodies in multiple myeloma: mechanisms of action and modes of resistance. Front Immunol. 2018;9:2134. doi:10.3389/fimmu.2018.02134

\section{Publish your work in this journal}

ImmunoTargets and Therapy is an international, peer-reviewed open access journal focusing on the immunological basis of diseases, potential targets for immune based therapy and treatment protocols employed to improve patient management. Basic immunology and physiology of the immune system in health, and disease will be also covered. In addition, the journal will focus on the impact of management

Submit your manuscript here: http://www.dovepress.com/immunotargets-and-therapy-journal programs and new therapeutic agents and protocols on patient perspectives such as quality of life, adherence and satisfaction. The manuscript management system is completely online and includes a very quick and fair peer-review system, which is all easy to use. Visit http://www.dovepress.com/testimonials.php to read real quotes from published authors. 